\title{
NECESSIDADES DA FAMÍLIA DO PACIENTE CRÍTICO EM TERMINALIDADE DE VIDA: REVISÃO INTEGRATIVA
}

\section{CRITICAL PATIENT'S FAMILY NEEDS IN TERMINALITY OF LIFE: INTEGRATIVE REVIEW}

\author{
Tábata de Cavatá Souza ${ }^{1} *$ Enaura Helena Brandão Chaves $^{2} *$ João Lucas Campos de Oliveira ${ }^{3}$ \\ Lisiane Nunes Aldabe ${ }^{4} *$ Aline dos Santos Duarte ${ }^{5} *$ Bibiana Fernandes Trevisan $^{6} *$ Mari Angela $^{2}$ \\ Victoria Lourenci Alves ${ }^{7}$ * Rodrigo D’Ávila Lauer ${ }^{8}$
}

\begin{abstract}
RESUMO
Objetivo: identificar na literatura científica as principais necessidades da família do paciente crítico terminal. Método: revisão integrativa realizada de acordo com as seis etapas propostas. As buscas foram realizadas nas bases de dados SciELO, LILACS e PubMed, sendo incluídos artigos publicados entre 2010 e março de 2021. Resultados: do total da amostra analisada (n=6), destacaram-se os ensaios clínicos $(50 \%)$ e pesquisas qualitativas (33\%). Foram evidenciadas diferentes necessidades dos familiares de pacientes críticos na terminalidade, com destaque para as necessidades de comunicação e as necessidades emocionais, espirituais, psicológicas e sociais. Considerações finais: a literatura científica aponta que a família do paciente em terminalidade de vida no âmbito da terapia intensiva apresenta uma diversidade de necessidades, e que o investimento na melhor comunicação com a equipe clínica durante o período de hospitalização na Unidade de Terapia Intensiva é uma premência. Percebe-se a importância da integração da equipe de saúde no contexto de terminalidade para auxiliar a família nesse momento delicado, corroborando para uma resposta adequada de um plano terapêutico. O trabalho interdisciplinar é uma alternativa, visto que as necessidades são de ordens diversas e não se limitam à alta densidade tecnológica comum a terapia intensiva.
\end{abstract}

Palavras-chave: Cuidados Críticos; Cuidados Paliativos na Terminalidade da Vida; Família; Enfermagem; Unidades de Terapia Intensiva.

\begin{abstract}
Objective: to identify in the scientific literature the main needs of the terminally critical patient's family. Method: integrative review carried out according to the six proposed steps. Searches were performed in the SciELO, LILACS and PubMed databases, including articles published between 2010 and March 2021. Results: from the total sample analyzed $(n=6)$, clinical trials $(50 \%)$ and research stood out qualitative (33\%). Different needs of families of critically ill patients in terminality were highlighted, with emphasis on communication needs and emotional, spiritual, psychological and social needs. Final considerations: the scientific literature indicates that the family of the terminally ill patient in the intensive care setting has a variety of needs, and that investing in better communication with the clinical team during the period of hospitalization in the Intensive Care Unit is a urgency. The importance of integrating the health team in the context of terminality to help the family in this delicate moment is perceived, supporting an adequate response to a therapeutic plan. Interdisciplinary work is an alternative, as the needs are of different orders and are not limited to the high technological density common to intensive care.
\end{abstract}

Keywords: Critical Care; Hospice Care; Family; Nursing; Intensive Care Units.

\footnotetext{
${ }^{1}$ Hospital de Clínicas de Porto Alegre. Porto Alegre, Rio Grande do Sul, Brasil. ORCID: 0000-0002-7758-218X.

${ }^{2}$ Universidade Federal do Rio Grande do Sul. Porto Alegre, Rio Grande do Sul, Brasil. ORCID: 0000-0001-8841-3624.

${ }^{3}$ Universidade Federal do Rio Grande do Sul. Porto Alegre, Rio Grande do Sul, Brasil. ORCID: 0000-0002-1822-2360.

${ }^{4}$ Hospital de Clínicas de Porto Alegre. Porto Alegre, Rio Grande do Sul, Brasil. ORCID: 0000-0001-9674-4634.

${ }^{5}$ Hospital de Clínicas de Porto Alegre. Porto Alegre, Rio Grande do Sul, Brasil. ORCID: 0000-0002-5357-1179.

${ }^{6}$ Hospital de Clínicas de Porto Alegre. Porto Alegre, Rio Grande do Sul, Brasil. ORCID: 0000-0002-9028-8073.

${ }^{7}$ Hospital de Clínicas de Porto Alegre. Porto Alegre, Rio Grande do Sul, Brasil. ORCID: 0000-0002-2297-416X.

${ }^{8}$ Hospital de Clínicas de Porto Alegre. Porto Alegre, Rio Grande do Sul, Brasil. ORCID: 0000-0002-8260-3766.
} 


\section{INTRODUÇÃO}

As questões sobre a terminalidade em terapia intensiva estão ligadas à limitação terapêutica e humanização, isso porque a equipe da Unidade de Terapia Intensiva (UTI), em geral, é treinada para salvar vidas, utilizando todos os recursos e tecnologias disponíveis para que essa meta seja atingida. Desta forma, para atender todas as necessidades de um paciente terminal, que vão muito além daquelas de ordem biológica, é necessária organização da dinâmica da unidade e capacitação constante da equipe assistencial $^{(1)}$.

O processo de morrer na UTI ainda está relacionado ao sofrimento e à dor de pacientes e suas famílias, muitas vezes ligadas ao ambiente mais hostil e estigmatizado que é a $\mathrm{UTI}^{(2)}$. Durante a proximidade da morte, o uso de tecnologias complexas e, principalmente, a pouca ou falha na comunicação entre profissionais, pacientes e familiares, evidenciam essa relação. Muitos familiares de pacientes internados em UTIs não têm uma boa compreensão das discussões sobre prognósticos e frequentemente têm expectativas distorcidas quanto à sobrevida, estado funcional e qualidade de vida dos seus entes queridos ${ }^{(3)}$.

Embora as diretrizes de sociedades profissionais recomendem estratégias para amparar as famílias de pacientes gravemente enfermos, evidências sugerem que muitas famílias podem se beneficiar desse tipo de apoio, mas não o recebem ${ }^{(4)}$. Os motivos para essa falha assistencial se relacionam comumente a falta de comunicação efetiva, suporte psicológico inadequado aos familiares e o despreparo da equipe de UTI em lidar com a terminalidade, o que traz como consequência para esses profissionais a sensação de fracasso frente a missão de curar o doente, tornando-se uma barreira no cuidado humanizado prestado pela equipe de saúde ${ }^{(5-6)}$.

Compreender a humanização em ambientes de cuidados complexos significa acolher a dinamicidade da organização destas unidades, gestão do trabalho dos profissionais e atendimento aos usuários, uma vez que as dinâmicas entre esses atores (re)alimentam as relações/interações humanas e profissionais que permeiam o cuidado ${ }^{(3)}$.

É necessário conhecer as diversas dificuldades enfrentadas pela família do paciente crítico terminal frente a situação atual, seja no âmbito psicossocial, econômico, espiritual e na comunicação com a equipe de saúde $^{(7)}$. Considerando o processo de terminalidade como uma etapa de difícil aceitação, o apontamento dessas necessidades serve para esclarecimentos de prognósticos, além de que os familiares possam externar seus anseios e medos, sentindo-se amparados pela equipe assistente ${ }^{(8)}$.

Uma vez apontado que ainda há necessidade de incremento no processo de 
terminalidade em UTI, e que a identificação das necessidades de famílias nesse processo pode contribuir para práticas de cuidado mais humanizadas e aproximadas do cuidado integral, o presente estudo objetivou identificar na literatura científica as principais necessidades da família do paciente crítico terminal.

\section{MÉTODO}

Trata-se de uma revisão integrativa, a qual foi desenvolvida em respeito às seis etapas propostas pelo referencial eleito: (1) elaboração da pergunta norteadora; (2) estabelecimento de critérios para inclusão/exclusão e busca/amostragem da literatura; (3) coleta de dados e categorização de informações; (4) avaliação crítica dos estudos incluídos; (5) interpretação dos resultados; e (6) apresentação da revisão integrativa, com a síntese do conhecimento ${ }^{(9)}$.

$1^{\mathrm{a}}$ etapa - o desenvolvimento desta revisão se deu por meio da formulação da seguinte questão norteadora: quais são as principais necessidades demandadas pela família do paciente adulto em terminalidade de vida internado em Unidade de Terapia Intensiva verificadas na literatura científica?

$2^{\mathrm{a}}$ etapa - a busca pelos artigos foi realizada nas seguintes bases de dados: Literatura Latino-Americana e do Caribe em Ciências da Saúde (LILACS), Scientific

\section{Eletronic Library Online (SciELO) e} MedLine, via PubMed.

Os descritores utilizados foram: “cuidados críticos" (critical care), "cuidados paliativos na terminalidade da vida" (hospice care) e "família" (family). Foram utilizadas combinações entre os descritores "cuidados críticos" AND “critical care" AND "family".

A busca na literatura ocorreu em abril de 2021. Os critérios de inclusão foram: artigos publicados entre 2010 e março de 2021; artigos com resumo e texto na íntegra, disponíveis gratuitamente nas bases de dados online; e escritos em inglês, espanhol ou português. Os critérios de exclusão foram estudos que não respondessem à pergunta de pesquisa; teses, dissertações e editoriais, além de publicações classificadas como artigos de revisão bibliográfica de qualquer natureza.

$3^{\mathrm{a}}$ etapa - com a busca de dados realizada, foram lidos os resumos de todos os textos recrutados $(n=37)$. Pela aplicação dos critérios de elegibilidade, seis artigos integraram a revisão. Destes, foram extraídas e sumarizadas as seguintes informações: título do artigo, periódico e ano de publicação, base de dados da qual o artigo foi recrutado, país de origem do estudo, tipo de estudo e conclusões. Os artigos foram identificados aleatoriamente em numerais romanos.

$4^{\mathrm{a}}$ etapa - a avaliação dos artigos selecionados se deu de forma interpretativa, com o intuito de extrair dos textos quais eram as principais necessidades dos familiares de 
pacientes terminais assistidos no contexto de UTI. Essas informações foram sumarizadas a fim de sistematizar o conhecimento.

$$
5^{\mathrm{a}} \text { etapa - a interpretação dos achados }
$$
ocorreu na discussão, atrelando os dados levantados/extraídos nas etapas anteriores com a literatura pertinente e as inferências autorais.

$6^{\mathrm{a}}$ etapa - a síntese do conhecimento se deu de forma ilustrativa, com uso de quadros sinópticos que sumarizam as informações extraídas na revisão integrativa.

Destaca-se que este estudo não fere os princípios éticos em pesquisa envolvendo seres humanos, por se tratar de uma pesquisa secundária. Todos os artigos de pesquisa primária tiveram as questões éticas cabíveis verificadas à sua leitura.

\section{RESULTADOS}

Utilizando os descritores citados, foram encontrados 12 artigos no SciELO, nenhum no LILACS e 25 no PubMed, totalizando 37 artigos nas bases de dados. Após leitura rigorosa, 06 artigos se enquadraram dentro da questão em estudo para análise e síntese do conhecimento (Quadro 1).

Quadro 1 - Relação dos artigos selecionados de acordo com título, periódico, ano, base de dados, origem do estudo, tipo de estudo e conclusões

\begin{tabular}{|c|c|c|c|c|c|}
\hline Código e título & $\begin{array}{c}\text { Periódico } \\
\text { e ano }\end{array}$ & $\begin{array}{c}\text { Base de } \\
\text { dados }\end{array}$ & $\begin{array}{c}\text { Origem } \\
\text { do estudo }\end{array}$ & Tipo de estudo & Conclusões \\
\hline $\begin{array}{l}\text { I) A brief intervention } \\
\text { for preparing ICU } \\
\text { families to be proxies: a } \\
\text { phase I study }{ }^{(10)}\end{array}$ & $\begin{array}{c}\text { PLoS One } \\
2017\end{array}$ & SciELO & $\begin{array}{l}\text { Estados } \\
\text { Unidos }\end{array}$ & $\begin{array}{l}\text { Ensaio clínico } \\
\text { com } 122 \\
\text { representantes } \\
\text { da saúde e } \\
111 \text { pacientes }\end{array}$ & $\begin{array}{l}\text { Considerada } \\
\text { positiva essa } \\
\text { intervenção de } \\
\text { comunicação da } \\
\text { família com } \\
\text { equipe clínica } \\
\end{array}$ \\
\hline $\begin{array}{l}\text { II) Grupo de suporte } \\
\text { como estratégia } \\
\text { para acolhimento de } \\
\text { familiares de pacientes } \\
\text { em Unidade de Terapia } \\
\text { Intensiva }{ }^{(11)}\end{array}$ & $\begin{array}{l}\text { Revista da } \\
\text { Escola de } \\
\text { Enfermagem } \\
\text { da USP } \\
2010\end{array}$ & SciELO & Brasil & $\begin{array}{c}\text { Pesquisa } \\
\text { descritiva com } \\
\text { abordagem } \\
\text { qualitativa, do tipo } \\
\text { convergente } \\
\text { assistencial com } \\
51 \text { participantes }\end{array}$ & $\begin{array}{l}\text { Recomenda-se } \\
\text { uma reflexão } \\
\text { sobre a } \\
\text { reorganização da } \\
\text { prática assistencial } \\
\text { para inclusão de } \\
\text { "grupo de suporte" } \\
\text { para suprir as } \\
\text { necessidades } \\
\text { dos familiares }\end{array}$ \\
\hline $\begin{array}{l}\text { III) A morte em cena na } \\
\text { UTI: a família diante da } \\
\text { terminalidade }{ }^{(12)}\end{array}$ & $\begin{array}{l}\text { Trends in } \\
\text { Psychology } \\
2017\end{array}$ & SciELO & Brasil & $\begin{array}{c}\text { Pesquisa clínico- } \\
\text { qualitativa com } 6 \\
\text { participantes }\end{array}$ & $\begin{array}{l}\text { Enfatizou-se o } \\
\text { comportamento } \\
\text { resiliente dos } \\
\text { familiares sobre } \\
\text { a terminalidade } \\
\end{array}$ \\
\hline
\end{tabular}




\begin{tabular}{|c|c|c|c|c|c|}
\hline $\begin{array}{l}\text { IV) A novel Family } \\
\text { Dignity Intervention } \\
\text { (FDI) for enhancing } \\
\text { and informing holistic } \\
\text { palliative care in Asia: } \\
\text { study protocol for } \\
\text { a randomized } \\
\text { controlled trial }^{(13)}\end{array}$ & $\begin{array}{l}\text { Trials } \\
2017\end{array}$ & PubMed & Cingapura & $\begin{array}{l}\text { Ensaio clínico } \\
\text { controlado } \\
\text { randomizado } \\
\text { aberto com } 126 \\
\text { participantes }\end{array}$ & $\begin{array}{l}\text { Abordou cuidados } \\
\text { psico-espirituais } \\
\text { a pacientes e } \\
\text { familiares que } \\
\text { enfrentam a } \\
\text { mortalidade } \\
\text { com sucesso }\end{array}$ \\
\hline $\begin{array}{l}\text { V) A randomized trial } \\
\text { of a family-support } \\
\text { intervention in } \\
\text { Intensive Care Units }\end{array}$ & $\begin{array}{l}\text { New } \\
\text { England } \\
\text { Journal of } \\
\text { Medicine } \\
2018\end{array}$ & PubMed & $\begin{array}{l}\text { Estados } \\
\text { Unidos }\end{array}$ & $\begin{array}{l}\text { Ensaio clínico } \\
\text { randomizado em } \\
\text { cluster envolvendo } \\
\text { pacientes com alto } \\
\text { risco de morte e } \\
\text { seus cuidadores } \\
\text { em cinco UTIs }\end{array}$ & $\begin{array}{c}\text { Apresentou } \\
\text { avaliação } \\
\text { satisfatória sobre } \\
\text { a qualidade da } \\
\text { comunicação da } \\
\text { equipe de saúde } \\
\text { e do paciente e } \\
\text { da família }\end{array}$ \\
\hline $\begin{array}{l}\text { VI) Shared decision- } \\
\text { making in end-stage } \\
\text { renal disease: a protocol } \\
\text { for a multi-center study } \\
\text { of a communication } \\
\text { intervention to improve } \\
\text { end-of-life care for } \\
\text { dialysis patients }\end{array}$ & $\begin{array}{c}\text { BMC } \\
\text { Palliative } \\
\text { Care } \\
2015\end{array}$ & PubMed & $\begin{array}{l}\text { Estados } \\
\text { Unidos }\end{array}$ & $\begin{array}{l}\text { Estudo de coorte } \\
\text { multicêntrico } \\
\text { que implantou } \\
\text { intervenção para } \\
\text { melhorar a } \\
\text { comunicação } \\
\text { de pacientes em } \\
\text { fim de vida em } \\
\text { hemodiálise }\end{array}$ & $\begin{array}{c}\text { Auxílio à } \\
\text { equipe de saúde } \\
\text { no planejamento } \\
\text { antecipados } \\
\text { de cuidados a } \\
\text { cuidadores e } \\
\text { pacientes no } \\
\text { final da vida }\end{array}$ \\
\hline
\end{tabular}

Fonte: Os autores

As principais necessidades encontradas pela família do paciente crítico terminal, estão descritas no Quadro 2.

Quadro 2 - Necessidades exploradas em cada artigo

\begin{tabular}{|c|c|}
\hline Artigos & Principais necessidades da família \\
\hline I, II, V e VI & Necessidades de comunicação \\
\hline II & Necessidades emocionais \\
\hline III, IV & Necessidades espirituais \\
\hline IV, V & Necessidades psicológicas \\
\hline III, IV & Necessidades sociais \\
\hline
\end{tabular}

Fonte: Os autores

\section{DISCUSSÃO}

Nos artigos I, II, V e VI a família do paciente crítico terminal relata que a comunicação é uma das principais necessidades a ser aprimorada. A comunicação é uma âncora do trabalho em saúde e é considerada uma tecnologia leve de cuidado $^{(16)}$. Ela precisa ser desenvolvida pelos trabalhadores para melhor articulação assistencial, e isso, inegavelmente, deve incorporar pacientes e familiares em todos os 
níveis de complexidade e fases da vida, incluindo a terminalidade ${ }^{(17)}$.

Estudo qualitativo sobre o enfrentamento da equipe assistencial ao cuidado do paciente crítico em terminalidade levantou três temas: barreiras acadêmicoculturais, relacionadas à orientação assistencial da UTI a pacientes e cuidadores e à falta de capacitação em cuidados em final de vida; barreiras arquitetônico-estruturais, relacionadas à falta de espaço e privacidade para o paciente e família nos últimos momentos da vida; e barreiras psicoemocionais, relacionadas ao uso do distanciamento emocional como estratégia aplicada pela equipe de enfermagem. Como possíveis soluções para estes desafios, os autores apontaram o treinamento da equipe de enfermagem sobre os cuidados em final de vida por meio da utilização de diretrizes ou protocolos e o desenvolvimento de estratégias de enfrentamento da assistência e comunicação efetiva aos familiares ${ }^{(18)}$.

Estudo multicêntrico, realizado para avaliar a satisfação da família em relação ao cuidado dos pacientes e de sua família, aplicou um questionário de satisfação entre janeiro de 2015 a fevereiro de 2016 em UTIs de três hospitais universitários terciários na Coreia do $\mathrm{Sul}^{(19)}$. Os achados apontaram os principais fatores que afetam a satisfação identificados por meio de análises quantitativas e qualitativas. As famílias relataram a menor satisfação com o ambiente da sala de espera, comunicação e gerenciamento da agitação do paciente. A decisão de não ressuscitar, a mortalidade na UTI e a cultura da UTI também estiveram associadas à satisfação da família com os cuidados intensivos. Neste sentido, os autores acreditam que os esforços de melhoria da qualidade assistencial devem ser direcionados na intervenção dos fatores que causam insatisfação da família do paciente crítico, e isso inclui melhoria nos processos de comunicação.

Já uma pesquisa de coorte realizada na Espanha avaliou a qualidade do atendimento clínico prestado a pacientes que morrem em $\mathrm{UTI}^{(20)}$. Os critérios de excelência em terapia intensiva foram avaliados por indicadores e medidas de qualidade, relacionados ao atendimento em fim de vida. Foram incluídos 282 pacientes de 15 UTIs espanholas. Quase todos os prontuários avaliaram tanto a capacidade de tomada de decisão do paciente quanto à comunicação do corpo clínica com os familiares. Apenas duas UTIs possuíam políticas abertas de visitação. A ausência de protocolo para a retirada de tratamentos de suporte de vida foi observada em 13 unidades. O estudo concluiu que a qualidade do cuidado ao final da vida na UTI participante precisa ser melhorado e que, apesar das lacunas existentes, pode ser desenhado um plano de melhoria gradual, adaptado à situação de cada hospital e UTI. 
Para determinar as perspectivas sobre como as informações prognósticas devem ser transmitidas em doenças críticas, realizou-se um estudo multicêntrico em três centros médicos acadêmicos na Califórnia, Pensilvânia e Washington ${ }^{(21)}$. Houve amplo apoio entre os familiares para as recomendações de especialistas existentes, incluindo divulgação de prognósticos verídicos, apoio emocional, adaptação da estratégia de divulgação às necessidades de cada família e verificação de compreensão. Além disso, os participantes adicionaram sugestões mais específicas, tal como o aprimoramento da comunicação sobre quadro de saúde do doente aos familiares. Além de transmitir estimativas de prognóstico, os médicos devem ajudar as famílias a "verem o prognóstico por si mesmas", mostrando imagens radiográficas das famílias e explicando o significado clínico das manifestações físicas de doença grave à beira do leito.

Para os participantes do estudo supracitado, os médicos devem conceituar a comunicação prognóstica como um processo interativo que começa com uma menção preliminar da possibilidade de morte no início da internação na UTI e se torna mais detalhado à medida que a situação clínica se desenvolve ${ }^{(21)}$.

Os artigos II, III, IV e V enfatizam a necessidade de auxílio psicológico, espiritual e emocional aos familiares no enfrentamento à internação e à terminalidade na terapia intensiva. A equipe de saúde deve acalmar, acolher e valorizar os sentimentos e expectativas do paciente e familiares ${ }^{(22)}$. Esse processo de acolhimento muitas vezes permeia o toque, a conversa e o saber ouvir, cuidados que, podem ser negligenciados devido à complexidade dos e a cultura dos cuidados intensivos.

Autores reforçam a importância da participação da psicologia no acolhimento e escuta dos familiares dos pacientes internados em UTI, visto que pela assistência dessa equipe tem-se a oportunidade de forma mais profissionalizada de falar sobre a terminalidade, podendo expressar o que sentem, como raiva, culpa, tristeza e estimulálos a dizerem um adeus apropriado. Com isso, as reações pós-óbito podem se tornar mais amenas e, consequentemente, favorecer uma melhor elaboração do luto ${ }^{(23)}$.

Outra categoria profissional indispensável no acolhimento e na humanização do cuidado em UTI é a enfermagem, pois essa equipe está em contato com o paciente diariamente, vivenciando medos e angústias dos pacientes e seus familiares $^{(24)}$. O enfermeiro, em especial, tem um papel fundamental no sentido de articulação da equipe interdisciplinar, uma vez reconhecida a sua característica/posição de gerente do cuidado. Pela experiência autoral, acredita-se que a articulação interprofissional favorecida pelo trabalho do 
enfermeiro tende a culminar em melhores resultados assistenciais, e também na maior participação e autonomia da família no processo de cuidado.

A autonomia dos familiares e dos próprios pacientes em UTI, apesar de não ter sido necessariamente expressa na síntese do conhecimento exposta, provavelmente permeia as necessidades identificadas, talvez em especial pela maior qualidade e/ou amplitude na comunicação dos profissionais intensivistas com os familiares. Este é um assunto importante a ser tratado no cenário dos cuidados críticos, até mesmo porque a possibilidade de se tornar um sujeito com poder de decisão sobre sua saúde já foi referida como frágil nesse âmbito ${ }^{(25)}$.

A respeito das necessidades espirituais identificadas, uma pesquisa descreveu o momento e a natureza dos encontros com capelães em UTIs ${ }^{(26)}$. Os achados apontam que as visitas do capelão são incomuns e geralmente ocorrem um pouco antes da morte entre os pacientes da UTI. A comunicação entre capelães e médicos é rara. O serviço de capelania é reservado principalmente para pacientes terminais e seus familiares, em vez de fornecer apoio espiritual proativo. Essas observações destacam a necessidade de compreender melhor os desafios e barreiras para o envolvimento ideal do capelão no atendimento ao paciente da UTI.

Recentemente, pesquisadoras brasileiras da área de enfermagem, com o objetivo de compreender a espiritualidade e a prática da eufemia vivenciada por profissionais de enfermagem no contexto hospitalar, concluíram que o os trabalhadores percebem a espiritualidade e a prática da eufemia como ferramenta motivacional à equipe para o enfrentamento das dificuldades vivenciadas no trabalho e o incremento da fé do paciente hospitalizado ${ }^{(27)}$. Somado a isso, outro estudo nacional realizado no sul do Brasil com pacientes hospitalizados referiu a espiritualidade, religiosidade e eufemia como uma tríade biopsicossocial, capaz de atribuir sentido, alicerce e bálsamo à vida humana ${ }^{(28)}$.

Em UTI, o respeito à proposição de condutas baseadas em valores espirituais do paciente crítico já foi referida por mais de $88 \%$ de uma amostra $(n=42)$ de enfermeiros, no estado de São Paulo, Brasil. As autoras deste estudo ainda acreditam que existe um tabu que permeia a dimensão espiritual e religiosa, o que dificulta o entendimento dos profissionais da saúde em conhecer a própria espiritualidade e como esta pode contribuir para a integralidade da assistência de enfermagem dispensada ao paciente crítico internado na $\mathrm{UTI}^{(29)}$.

Apesar da relevância das alusões expressas, destaca-se que o estudo antes referido se ancora sobre a espiritualidade dos pacientes e, com essa revisão, percebe-se que também há necessidade de se ampliar a visão sobre as necessidades espirituais dos familiares destes indivíduos. Essa ampliação, 
possivelmente, pode contribuir para a efetividade do cuidado e transposição desse cuidado ao ser doente, uma vez que ele faz parte de um meio social familiar.

Os estudos III e IV destacam a necessidade da rede de apoio social aos familiares e aos pacientes. Os autores de um estudo realizado em quatro UTIs dos Estados Unidos afirmam que, para tornar o processo de tomada de decisão familiar eficaz, é importante reconhecer e compreender os papéis informais que vários membros da família podem desempenhar no processo de tomada de decisão do fím da vida ${ }^{(30)}$. Os autores concluíram que os papéis informais da família refletem as diversas respostas às demandas de tomada de decisão da família, o que geralmente é uma situação nova e estressante. A identificação desses papéis pode ajudar os profissionais a compreenderem as funções de cada familiar além de orientar o desenvolvimento de estratégias para apoiar e facilitar o aumento da eficácia dos processos de tomada de decisão no momento do adoecimento e morte do ente querido.

É prudente assumir que existem limitações a serem declaradas a este estudo, as quais guardam relação, principalmente, à ausência de estratégias de busca mais avançadas e em maior número de bases de dados, além de a pesquisa nas bases ter sido feita por uma única pesquisadora. No entanto, acredita-se que o estudo contribui no sentido de definir com clareza algumas necessidades que são demandadas pelo familiar do doente terminal na UTI. Isso certamente pode suscitar debates e planejamento sobre ações para um cuidado mais sensível e integral nessa fase da vida, e que transponha as barreiras clínico-assistenciais do doente crítico. Por fim, sabe-se que é importante que pesquisas futuras sejam desenvolvidas sobre a importância da presença e da autonomia da família em situações de terminalidade.

\section{CONSIDERAÇÕES FINAIS}

Conclui-se que a literatura científica aponta que a família do paciente em terminalidade de vida no âmbito da terapia intensiva apresenta uma diversidade de necessidades. A síntese do conhecimento permitida por este estudo aponta que tais necessidades se vinculam a: necessidades de comunicação e necessidades emocionais, espirituais, psicológicas e sociais.

Ante o exposto, considerando que as necessidades são eminentemente de teor relacional, considera-se que o investimento na melhor comunicação com a equipe clínica durante o período de hospitalização na Unidade de Terapia Intensiva é uma premência. Nesse efetivo processo comunicativo, espera-se que a família seja acolhida, ouvida, e suas necessidades sistematizadas, a fim de serem sanadas e/ou atenuadas. 
Percebe-se a importância da integração da equipe de saúde no contexto de terminalidade para auxiliar a família nesse momento delicado, corroborando para uma resposta adequada de um plano terapêutico. $\mathrm{O}$ trabalho interdisciplinar é uma alternativa, visto que as necessidades são de ordens diversas e não se limitam à alta densidade tecnológica comum a terapia intensiva.

\section{REFERÊNCIAS}

1. Gulini JEHMB, Nascimento ERP, Moritz RD, Rosa LM, Silveira NR, Vargas MAO. A equipe da Unidade de Terapia Intensiva frente ao cuidado paliativo: discurso do sujeito coletivo. Rev Esc Enferm USP [Internet]. 2017 [acesso em 01 jul. 2021];51:e03221. Disponível

em: http://dx.doi.org/10.1590/S1980220X2016041703221

2. Costa TF, Morais JMD, Oliveira AMM, Rodrigues FA, Montenegro SMSL, Holanda AR. Terminalidade e cuidados paliativos em UTI: discurso dos técnicos de enfermagem. Rev Enferm UFPE on line [Internet]. 2014 [acesso em 01 jul. 2021];8(5):1157-63. Disponível em: https://periodicos.ufpe.br/revistas/revistaenfer magem/article/view/9794/9952

3. Nascimento EA, Lima LNF, Pereira CS, Fonseca SCT, Silva DO, Neves AF, et al. As dificuldades da equipe de enfermagem frente à assistência humanizada na unidade de terapia intensiva. Braz J of Dev [Internet]. 2021 [acesso em 01 jul. 2021];7(1):17262-72. Disponível em: https://www.brazilianjournals.com/index.php/ BRJD/article/view/24946/19890

4. Penrod JD, Pronovost PJ, Livote EE, Puntillo KA, Walker AS, Wallenstein S, et al. Meeting standards of high-quality intensive care unit palliative care: clinical performance and predictors. Crit Care Med [Internet]. 2012 [acesso em 01 jul. 2021];40(4):1105-12. Disponível em: https://www.ncbi.nlm.nih.gov/pmc/articles/P MC3307856/.

5. Takahashi CB, Contrin LM, Beccaria LM, Goudinho MV, Pereira RAM. Morte: percepção e sentimentos de acadêmicos de enfermagem. Arq Ciênc Saúde [Internet]. 2008 [acesso em 01 jul. 2021];15(3):132-8. Disponível em: https://repositorioracs.famerp.br/racs_ol/vol-15-3/IDN295.pdf

6. Luiz FF, Caregnato RCA, Costa, MR. Humanization in the Intensive Care: perception of family and healthcare professionals. Rev Bras Enferm [Internet]. 2017 [acesso em 01 jul. 2021];70(5):1040-7. Disponível em: http://dx.doi.org/10.1590/0034-7167-20160281

7. Santos QN. Estratégia de enfrentamento (coping) da família ante um membro familiar hospitalizado: uma revisão de literatura brasileira. Mudanças [Internet]. 2013 [acesso em 01 jul. 2021];21(2):40-7. Disponível em: http://dx.doi.org/10.15603/21761019/mud.v21n2p40-47

8. Goi MG, Oliveira DR. Produção do conhecimento de enfermagem acerca de cuidados paliativos: revisão narrativa. Rev Contexto Saúde [Internet]. 2018 [acesso em 01 jul. 2021];18(34):20-6. Disponível em: http://dx.doi.org/10.21527/2176-

7114.2018.34.20-26

9. Mendes KDS, Silveira RCCP, Galvão CM. Revisão integrativa: método de pesquisa para a incorporação de evidências na saúde e na enfermagem. Texto Contexto Enferm [Internet]. 2008 [acesso em 01 jul. 2021];17(4):758-64. Disponível em: http://dx.doi.org/10.1590/S010407072008000400018

10. Turnbull AE, Chessare CM, Coffin RK, Needham DM. A brief intervention for preparing ICU families to be proxies: a phase I study. PLoS One [Internet]. 2017 [acesso em 01 jul. 2021];12(10):e0185483. Disponível em: 
https://journals.plos.org/plosone/article?id=10 .1371/journal.pone.0185483

11. Oliveira LMAC, Medeiros M, Barbosa MA, Siqueira KM, Oliveira PMC, Munari DB. Grupo de suporte como estratégia para acolhimento de familiares de pacientes em Unidade de Terapia Intensiva. Rev Esc Enferm USP [Internet]. 2010 [acesso em 01 jul. 2021];44(2):429-36. Disponível em: http://dx.doi.org/10.1590/S008062342010000200027

12. Monteiro MC, Magalhães AS, Machado RN. A morte em cena na UTI: a família diante da terminalidade. Trends Psychol [Internet]. 2017 [acesso em 01 jul. 2021];25(3):1285-99. Disponível em: http://dx.doi.org/10.9788/TP2017.3-17Pt

13. Ho AHY, Car J, Ho MR, Tan-Ho G, Choo PY, Patinadan PV, et al. A novel Family Dignity Intervention (FDI) for enhancing and informing holistic palliative care in Asia: study protocol for a randomized controlled trial. Trials [Internet]. 2017 [acesso em 01 jul. 2021];18(587):1-12. Disponível em: https://trialsjournal.biomedcentral.com/article s/10.1186/s13063-017-2325-5

14. White DB, Angus DC, Shields AM, Buddadhumaruk P, Pidro C, Paner C, et al. A randomized trial of a family-support intervention in Intensive Care Units. N Engl J Med [Internet]. 2018 [acesso em 01 jul. 2021];378:2365-75. Disponível em: https://www.nejm.org/doi/full/10.1056/NEJM oa1802637

15. Eneanya ND, Goff SL, Martinez T, Gutierrez N, Klingensmith J, Griffith JL, et al. Shared decision-making in end-stage renal disease: a protocol for a multi-center study of a communication intervention to improve end-of-life care for dialysis patients. BMC Palliat Care [Internet]. 2015 [acesso em 01 jul. 2021];14(30):1-7. Disponível em: https://bmcpalliatcare.biomedcentral.com/arti cles/10.1186/s12904-015-0027-x

16. Torres GMC, Figueiredo IDT, Cândido JAB, Morais APP, Almeida MI. O emprego das tecnologias leves no cuidado ao hipertenso na Estratégia Saúde da Família.
Esc Anna Nery [Internet]. 2018 [acesso em 01 jul. 2021];22(3):e20170169. Disponível em: http://dx.doi.org/10.1590/2177-9465-EAN2017-0169

17. Almeida Q, Fófano GA. Tecnologias leves aplicadas ao cuidado de enfermagem na unidade de terapia intensiva: uma revisão de literatura. HU Rev [Internet]. 2016 [acesso em 01 jul. 2021];42(3):191-6. Disponível em: https://periodicos.ufjf.br/index.php/hurevista/ article/view/2494/891

18. Velarde-García JF, Luengo-González R, González-Hervías R, González-Cervantes S, Álvarez-Embarba B, Palacios-Ceña D. Dificultades para ofrecer cuidados al final de la vida en las unidades de cuidados intensivos. La perspectiva de enfermería. Gac Sanit [Internet]. 2017 [acesso em 01 jul. 2021];31(4):299-304. Disponível em: http://dx.doi.org/10.1016/j.gaceta.2016.11.00 6

19. Min J, Kim Y, Lee JK, Lee H, Lee J, Kim $\mathrm{KS}$, et al. Survey of family satisfaction with intensive care units: a prospective multicenter study. Medicine (Baltimore) [Internet]. 2018 [acesso em 01 jul. 2021];97(32):e11809. Disponível em: https://www.ncbi.nlm.nih.gov/pmc/articles/P MC6133602/.

20. Girbau MB, Monedero P, Centeno C. El buen cuidado de pacientes que fallecen en unidades de cuidados intensivo en España: un estudio basado en indicadores internacionales de calidad asistencial. An Sist Sanit Navar. 2017;40(3):339-49.

21. Anderson WG, Cimino JW, Ernecoff NC, Ungar A, Shotsberger KJ, Pollice LA, et al. A multicenter study of key stakeholders' perspectives on communicating with surrogates about prognosis in intensive care units. Ann Am Thorac Soc [Internet]. 2015 [acesso em 01 jul. 2021];12(2):142-52. Disponível em: https://www.ncbi.nlm.nih.gov/pmc/articles/P MC4342839/.

22. Pott FS, Stahlhoefer T, Felix JVC, Meier MJ. Medidas de conforto e comunicação nas ações de cuidado de enfermagem ao paciente 
crítico. Rev Bras Enferm [Internet]. 2013 [acesso em 01 jul. 2021];66(2):174-9. Disponível

em: http://dx.doi.org/10.1590/S0034-

71672013000200004

23. Ferreira PD, Mendes TN. Família em UTI: importância do suporte psicológico diante da iminência de morte. Rev SBPH [Internet]. 2013 [acesso em 01 jul. 2021];16(1):88-112. Disponível em: http://pepsic.bvsalud.org/pdf/rsbph/v16n1/v16 n1a06.pdf

24. Bezerra JM, Fonseca IAC. Unidade de terapia intensiva adulto: percepção da equipe de enfermagem sobre o cuidado ao paciente grave. Rev Eletrônica Acervo Saúde [Internet]. 2019 [acesso em 01 jul. 2021];31:e1060. Disponível em: https://acervomais.com.br/index.php/saude/art icle/view/1060/675

25. Damion M, Moreira MC. Percepção do paciente sobre sua autonomia na Unidade de Terapia Intensiva. Contextos Clínic [Internet]. 2018 [acesso em 01 jul. 2021];11(3):386-96. Disponível em: http://pepsic.bvsalud.org/pdf/cclin/v11n3/v11 n3a10.pdf

26. Choi PJ, Curlin FA, Cox CE. "The patient is dying, please call the chaplain": the activities of chaplains in one medical center's intensive care units. J Pain Symptom Manage [Internet]. 2015 [acesso em 01 jul. 2021];50(4):501-6. Disponível em: https://www.jpsmjournal.com/article/S08853924(15)00245-6/fulltext

27. Maran E, Matsuda LM, Spigolon DN, Teston EF, Almeida ES, Silva PA, et al. Espiritualidade e prática da eufemia no ambiente de trabalho: percepções de uma equipe de enfermagem. Rev Bras Enferm
[Internet]. 2020 [acesso em 01 jul. 2021];73(Suppl 6):e20190707. Disponível em: http://dx.doi.org/10.1590/0034-71672019-0707

28. Camillo NRS, Matsuda LM, Maran E, Pini JS, Aveiro HEP, Labegalini CMG, et al. Perception of spirituality, religiosity, and euphemia in the light of hospitalized patients. Rev Rene [Internet]. 2021 [acesso em 01 jul. 2021];22:e62502. Disponível em: http://periodicos.ufc.br/rene/article/view/6250 2/196918

29. Tavares AL, Devezas AMLO, Reppetto MA, Santos LSC. Atenção do enfermeiro em relação a espiritualidade no cuidar do paciente em unidade de terapia intensiva. Rev Recien [Internet]. 2020 [acesso em 01 jul. 2021];10(30):62-7. Disponível em: https://www.recien.com.br/index.php/Recien/ article/view/362/pdf

30. Quinn JR, Schmitt M, Baggs JG, Norton SA, Dombeck MT, Sellers CR. Family members' informal roles in end-of-life decision making in adult intensive care units. Am J Crit Care [Internet]. 2012 [acesso em 01 jul. 2021];21(1):43-51. Disponível em: https://www.ncbi.nlm.nih.gov/pmc/articles/P MC3615559/.

\section{Autor correspondente}

Tábata de Cavatá Souza

RS 287, Km 30, Casa n 01 - Tabaí - Rio Grande do Sul - 95863-000

+55(51) 998814906

tabatasouza@hcpa.edu.br

Submissão: 2021-07-05

Aprovado: 2021-10-26 